"tmcs-centroids" — 2013/5/24 — 16:50 — page $67-\# 1$

\title{
Teaching centroids in theory and in practice
}

\author{
SzILÁRd ANDrÁs and CsabA TAmÁsI
}

In theory, theory and practice are the same. In practice, they are not. Albert Einstein

\begin{abstract}
The main aim of this paper is to present an inquiry-based professional development activity about the teaching of centroids and to highlight some common misconceptions related to centroids. The second aim is to emphasize a major hindering factor in planning inquiry based teaching/learning activities connected with abstract mathematical notions. Our basic problem was to determine the centroid of simple systems such as: systems of collinear points, arbitrary system of points, polygons, polygonal shapes. The only inconvenience was that we needed practical activities where students could validate their findings and calculations with simple tools. At this point we faced the following situation: we have an abstract definition for the centroid of a finite system of points, while in practice we don't even have such systems. The same is valid for geometric objects like triangles, polygons. In practice we have triangular objects, polygonal shapes (domains) and not triangles, polygons. Thus in practice for validating the centroid of a system formed by $4,5, \ldots$ points we also need the centroid of a polygonal shape, formed by an infinite number of points. We could use, of course, basic definitions, but our intention was to organize inquiry based learning activities, where students can understand fundamental concepts and properties before defining them.
\end{abstract}

Key words and phrases: centroid, centroid of point systems, centroid of polygons and polygonal shapes, inquiry-based teaching.

ZDM Subject Classification: G43, U63.

\section{Introduction, the tasks}

The use of inquiry-based learning into day-to-day teaching practice has become an important goal ([7], [4]). On the other hand the study of problems from 
"tmcs-centroids" — 2013/5/24 — 16:50 — page 68 - \#2

applications was traditionally an organic part of mathematics ([2]), and in the last decades the applied mathematics acquired a greater role inside mathematics, hence the applied mathematics has to have a similar role also in the teaching of mathematics and also in the assessment. Unfortunately there are many European countries in which neither the curricula, nor the assessment does reflect this. Moreover, in some countries (such as Romania) the existing curricula is tightly fitted as content and volume to the teacher-centered pedagogy, so it is a difficult problem to use inquiry-based learning in an effective way (strongly related to the content without skewing the principles of IBL). Our experience shows that if we want students to deeply understand mathematics and science not only as a collection of useful rules and results, but also as a basic human activity, then we need to use a more flexible structure in content construction, which allows to use students' questions and curiosity as driving force.

A real inquiry-based teaching needs specially designed teaching materials, autonomous teachers who can use them and also a group of open-minded students. In what follows we present a teaching material which was designed by the members of the SimpleX Association and was used during a professional development course organized by the project PRIMAS ${ }^{1}$ at the Babeş-Bolyai University. We tested this material with lower secondary school studens (in Băţanii Mari and Miercurea Ciuc), with upper secondary school students (in a summer camp at Bonţida and at the Márton Áron Highschool in Miercurea Ciuc), with pre-service teachers (at a special course at Babeş-Bolyai University) and with inservice teachers (at different workshops and professional development courses). We also present some problems we detected during the testing of this material. For a clear understanding in what follows we refer to these groups as:

- group A: 13-15 years old lower secondary school students;

- group B: 16-17 years old upper secondary school students;

- group C: 22 years old pre-service teachers;

- group D: in-service teachers.

Since we had 8 different activities (more than 24 hours of activity in total) with 3 different type of participants, we do not want to focus on a specific subgroup from an activity. We would like to give an overview of possible approaches at different levels and to emphasize also the mathematical content and some related misconceptions.

${ }^{1}$ Promoting Inquiry in Mathematics and Science Across Europe, http://www.primas-project. eu 


$$
\text { "tmcs-centroids" — 2013/5/24 — 16:50 — page } 69-\# 3
$$

Our basic problem is to determine the centroid of simple systems such as: systems of collinear points, arbitrary system of points, polygons, polygonal shapes. All groups have learnt about centroid of triangles at mathematics lessons, about the centroid of a solid at physics lessons. Moreover groups $B, C$ and $D$ learnt about centroid of polygons in general and teachers from group $D$ also have taught this lesson for their students. We were particulary interested in finding how this preliminary knowledge is used during a hands on activity. For this reason we did not provide any definition about these concepts before the activities, all the activities were built on the participants preconceptions.

In the first part of our activity we focused on the centroid of systems of points and in the second part we studied polygonal shapes. Participants were working in groups and for the first part each group had a set of weights (salt bags), a rod, a measuring tape, a small board with a nail and an electronic scale (see Figure 1).

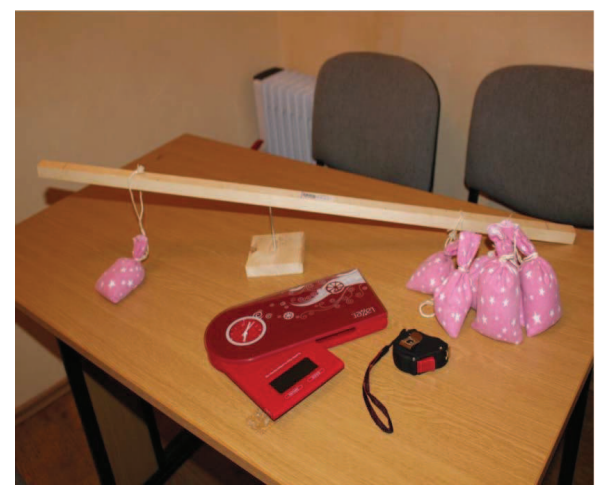

Figure 1. The tools for the activities

The groups had the following tasks for Part I:

- Put 2 (unequal) weights on the rod and calculate the centroid of the obtained system. Validate your calculations!

- Put a third weight on the rod and calculate the centroid of the system! Validate your calculations!

- Give a general method, which can be used for any finite number of weights on the rod!

For the second part each group had several polygonal shapes cut out of colour paper $\left(250 \mathrm{~g} / \mathrm{m}^{2}\right)$ and board. Part II consists of the following tasks: 
- Calculate or construct the centroid of the paper triangle! Validate your calculations!

- Calculate or construct the centroid of the paper polygon! Validate your calculations!

- Calculate or construct the centroid of the polygonal shapes made of board! Validate your calculations!

- Give a general method, which can be used for determining the centroid of any polygonal shape!

For the sake of simplicity we accepted that the validation should be in practice, so in each case the nail has to support the system in an equilibrium if it is positioned in the centroid.

Comment 1. The problem itself is a classical one, but it is a little bit tricky because the students have to figure out that they can not neglect the rod (in part I) and the board (in part II), so the real problem is how to handle the weight of the rod (or board). It is also important to mention that students had no previous knowledge about centroid of planar shapes, they had only learned about the centroid of a triangle and of a quadrilateral. Moreover, the language they use is misleading for several reasons:

- at school students learn (in theory) about the centroid of triangles, quadrilaterals and polygons, without making a difference between polygons and polygonal shapes;

- students often use other syntagma in which the concept of polygon and polygonal shape are mixed up without creating misunderstanding, such a syntagma is the area of a polygon;

- as we will see in the sequel, in the case of triangles the centroid of the homogeneous triangular shape is the same as the centroid of the system formed by the vertices of the triangle (having equal weights).

The main purpose of the activities, behind the formulated tasks, was to discover and understand the following lemma of Archimedes (see [1]): If an object is divided into two smaller objects, the centroid of the compound object lies on the line segment joining the centroids of the two smaller objects. 


$$
\text { "tmcs-centroids" — 2013/5/24 — 16:50 — page } 71-\# 5
$$

\section{Centroid of system of points}

The first task proved to be tricky because all our groups (from lower secondary students to teachers) calculated the centroid of a system with two points $A$ and $B$ (situated at the ends of the rod) and having mass $m_{1}$ and $m_{2}$. This is the point $G$ with the properties

$$
m_{1} \cdot A G=m_{2} \cdot B G
$$

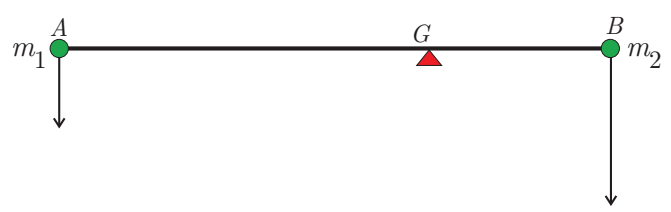

Figure 2. The first idea

From this we obtain

$$
A G=\frac{m_{2}}{m_{1}+m_{2}} d \quad \text { and } \quad B G=\frac{m_{1}}{m_{1}+m_{2}} d,
$$

where $d$ is the length of the rod (the distance $A B$ ). But as the proof of the pudding is in the eating, this result needs to be validated by a practical test, and in all the cases this test failed (the system supported in $G$ was not in equilibrium). All groups had to determine the cause of this practical fact. Lower secondary school students thought that there is something wrong with relation (1) or with their calculations. Students from upper secondary school thought that the rod is not homogeneous, or the supporting surface (the nail) is too small. University students and teachers, thought that the rod is not homogeneous, or the fact that $A$ and $B$ are not exactly at the end of the rod (because the weights were hanged up) can cause this problem.

Some of them realized at the beginning of the activity, that somehow they have to deal also with the rod, but during the activity they forgot their initial idea and they got stuck in analyzing their calculations. After a few minutes (15 for lower secondary school students, 12-15 for upper secondary school students and 9-10 minutes for teachers) some groups measured the weight of the rod and they started to think about how to use this data to correct their initial answer. The following ideas came up during the activities (we listed in parenthesis all type of groups were the idea appeared): 


$$
\text { "tmcs-centroids" — 2013/5/24 - 16:50 — page } 72 \text { — \#6 }
$$

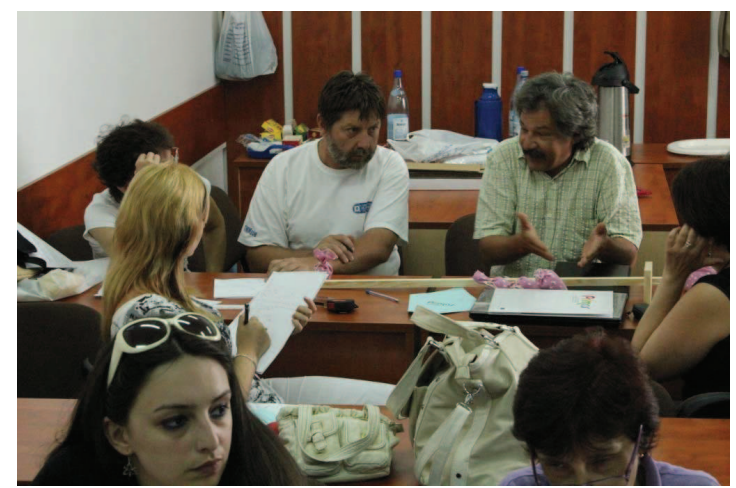

Figure 3. Something is wrong with the rod!

a) divide the rod into two parts having weights proportional to the initial weights and add these weights to the initial weights $(\mathrm{A}, \mathrm{B}, \mathrm{C}, \mathrm{D})$;

b) divide the rod into small, but congruent pieces, calculate the weight of a small piece and add some small pieces to the weight in $A$, the rest of the small pieces to the weight in $B(\mathrm{C}, \mathrm{D})$;

c) distribute the weight of the rod evenly between $A$ and $B(\mathrm{~A})$;

d) determine the centroid experimentally (by a trial and error method using the nail) and distribute the weight of the rod proportional to the distances of the experimented centroid from the endpoints $(\mathrm{A}, \mathrm{D})$;

e) calculate the centroid $G$ of the system formed by the points $A$ and $B$ with masses $m_{1}$ and $m_{2}$ and consider the midpoint of the segment $O G$, where $O$ is the midpoint of the $\operatorname{rod}(\mathrm{A}, \mathrm{B}, \mathrm{C}, \mathrm{D})$;

f) calculate the position of $G$ as presented above, calculate the weight of the rod's parts $A G$ and $B G$, distribute the difference between the weights of these parts evenly to $A$ and $B$ and repeat the procedure (C,D);

g) denote by $x$ the length of $A G$, where $G$ is the centroid of the system and consider two more weights concentrated at the midpoint of $A G$, respectively $B G$, proportional to the lengths of $A G$ and $B G(\mathrm{~A}, \mathrm{~B}, \mathrm{C}, \mathrm{D})$

h) concentrate the mass of the rod into it's midpoint $O$ and calculate the centroid of the system $G O$, where $G$ is the center of mass for the system $\{A, B\}$ having masses $m_{1}, m_{2}(\mathrm{~B}, \mathrm{D})$ 
i) The moment of the weight forces $F_{1}, F_{2}$ and $F$ has to equal to the moment of the force $F_{1}+F_{2}+F$ localized at the centroid (B,D).

At our activities the main strategy was to discuss the incorrect ideas first and then to give the opportunity for the groups to formulate other ideas. The correctness of the ideas was validated in practice (see Figure 8.). Moreover we compared the details of calculations belonging to the different ideas. This seemed to be important for several aspects:

a) to understand that behind every formal calculation there are some ideas and these ideas usually needs to be clarified before starting calculations;

b) to emphasize that there is no unique good way of solving the problem;

c) to compare the volume and complexity of calculations belonging to different ideas;

d) when working with several masses, this comparison was necessary in order to emphasize the fact that we can group the masses in any possible way.

We analyzed all ideas formulated by the groups using a discussion among groups. When working with teachers, we presented some of the ideas from previous activities (when we were working with students) and we asked them to analyze these as students' answers and to develop a strategy for helping students in understanding their mistakes and in finding a possible good solution. A good example of such a task is to explain why $c$ ) gives the correct answer. This task created a very unpleasant situation for teachers: they saw that the idea is working, the result is correct, but they were not able to give a quick (10 minutes) explanation.

When we worked with students, we started with eliminating some ideas by experimenting them (calculating the centroid and validating the calculations in practice) and by finding arguments against them. As an example for $c$ ) we asked why to distribute evenly the weight between $A$ and $B$ ? Find a case when this idea would give a correct answer! The answer was that if $m_{1}=m_{2}$, then this idea is working, the centroid is the midpoint of the rod. This led the groups to focus on a possible strategy for the distribution of the weight of the rod. From this we obtained $a), d$ ) and $f$ ). For $e$ ) students also gave a special case when this idea is working, this is the case when $m_{1}+m_{2}$ is equal to the mass of the rod, but they dropped the idea because the result obtained was not correct in practice. From this special case some groups developed $h$ ). b) and $g$ ) were also formulated from studying the break-up of the weight of the rod. On the other hand, $a$ ) gives the same point as the initial incorrect reasoning, $d$ ) needs the experimental determination of the centroid (and this could be unavailable if the 
system is too heavy and the centroid is needed in order to lift up the system), so only $b), c), f), g), h$ ) and $i$ ) needs a deeper analysis. In what follows we reproduce the calculations for these cases. First we discuss $g$ ), which was discovered in all groups, after this we detail the solutions given by upper secondary school students and teachers. After this we follow the calculations for $c$ ), which appeared only with lower secondary school students (and seemed unnaturally for teachers) and finally we discuss the ideas that didn't appear in groups A and B.

$g$ ) The mass of the piece $A G$ is $\frac{x}{d} m$, while the mass of piece $B G$ is $\frac{d-x}{d} m$, where $m$ is the mass of the rod. The pieces $A G$ and $B G$ are homogeneous, so their weight can be considered as acting in there midpoint (see Figure 7). From these considerations we obtain the following equation:

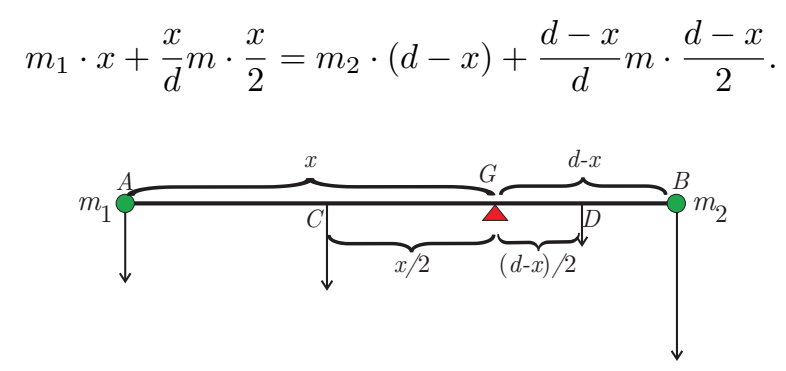

Figure 4. A promising choose of variables

This relation implies

$$
A G=\frac{m_{2}+\frac{m}{2}}{m_{1}+m_{2}+m} d \quad \text { and } \quad B G=\frac{m_{1}+\frac{m}{2}}{m_{1}+m_{2}+m} d .
$$

h) Consider $G_{1}$ the centroid of the system $\{A, B\}$ with masses $m_{1}$ and $m_{2}$ and concentrate the mass $m_{1}+m_{2}$ into $G_{1}$. Suppose $m_{2}>m_{1}$. In this case $G_{1} B=\frac{m_{1}}{m_{1}+m_{2}} d$. The rod is homogeneous, so its mass $m$ can be concentrated into it's midpoint $O$. If $G$ is the centroid of the system $\left\{O, G_{1}\right\}$ with masses $m$ respectively $m_{1}+m_{2}$, then according to our first idea we have:

$$
G G_{1}=\frac{m}{m_{1}+m_{2}+m} O G_{1}=\frac{m}{m_{1}+m_{2}+m} \frac{m_{2}-m_{1}}{m_{1}+m_{2}} \frac{d}{2},
$$

because $O G_{1}=\frac{d}{2}-G_{1} B=\frac{m_{2}-m_{1}}{m_{1}+m_{2}} \frac{d}{2}$.

But $B G=B G_{1}+G_{1} G$, so we obtain

$$
B G=\frac{m_{1}}{m_{1}+m_{2}} d+\frac{m}{m_{1}+m_{2}+m} \frac{m_{2}-m_{1}}{m_{1}+m_{2}} \frac{d}{2}=\frac{m_{1}+\frac{m}{2}}{m_{1}+m_{2}+m} d .
$$

This is the same result as using idea $g$ ). 


$$
\text { "tmcs-centroids" — 2013/5/24 - 16:50 — page } 75 \text { — \#9 }
$$

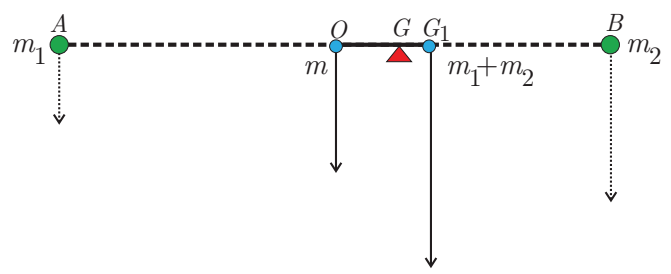

Figure 5. Grouping the weights

REMARK 1. For simplicity in what follows we denote by $X(u)$ the mass $u$ located at $X$.

REMARK 2. We obtain the same result if first we group $A\left(m_{1}\right)$ with $O(m)$ and then we calculate the centroid of the system $G_{2}\left(m_{1}+m\right), B\left(m_{2}\right)$, where $G_{2}$ is the centroid of the system $\left\{A\left(m_{1}\right), O(m)\right\}$.

i) Consider $X$ an arbitrary point outside the segment $A B$ such that $B \in(A X)$ and denote $B X=l$. The sum of the moments of the weight forces located in $A$, $B$ and the midpoint of $A B$ is $M=m_{1} \cdot(d+l)+m_{2} \cdot l+m \cdot\left(\frac{d}{2}+l\right)$. If the distance of the centroid from $B$ is $x$, then we must have

$$
M=\left(m_{1}+m_{2}+m\right)(x+l)
$$

so we obtain

$$
B G=\frac{m_{1}+\frac{m}{2}}{m_{1}+m_{2}+m} d,
$$

which is the same result as in the case $c), g$ ) or $h$ ).

$c$ ) If we distribute the mass of the rod evenly, we have a system of two points ( $A$ and $B$ ) with masses $m_{1}+\frac{m}{2}$ and $m_{2}+\frac{m}{2}$ (see Figure 6 ). Repeating our first argument for the centroid $G$ of this system we obtain

$$
A G=\frac{m_{2}+\frac{m}{2}}{m_{1}+m_{2}+m} d \quad \text { and } \quad B G=\frac{m_{1}+\frac{m}{2}}{m_{1}+m_{2}+m} d,
$$

which is the same result as in the case $g$ ) or $h$ ).

$b)$ It is clear that we obtain only an approximation of the centroid and this approximation is depending on the size of the small pieces. Suppose we divide the rod into $n$ small pieces. The mass of each is $\frac{m}{n}$, where $m$ is the mass of the rod. If for the centroid $G$ we have $A G=\frac{k}{n} \cdot d$, for some integer $k \in\{1,2, \ldots, n\}$, and we consider that the mass of each small piece is concentrated in the furthest 


$$
\text { "tmcs-centroids" — 2013/5/24 — 16:50 — page } 76 \text { — \#10 }
$$

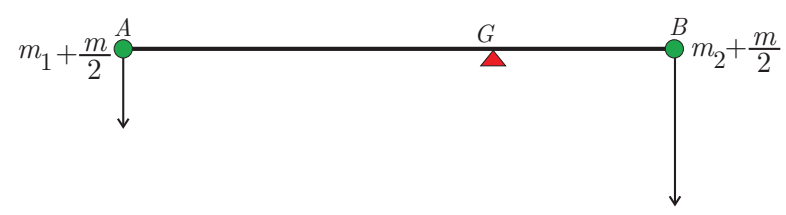

Figure 6. Distribute evenly the mass of the rod

endpoint of this piece with respect to $G$, then we obtain

$$
\begin{aligned}
\frac{m}{n} \cdot \frac{d}{n}+\frac{m}{n} \cdot \frac{2 d}{n}+\cdots & +\frac{m}{n} \cdot \frac{k d}{n}+m_{1} \frac{k d}{n} \\
& =\frac{m}{n} \cdot \frac{d}{n}+\frac{m}{n} \cdot \frac{2 d}{n}+\cdots+\frac{m}{n} \cdot \frac{(n-k) d}{n}+m_{2} \frac{(n-k) d}{n}
\end{aligned}
$$

From this relation expressing $\frac{k}{n}$, we get:

$$
\frac{k}{n}=\frac{\frac{m}{2}+m_{2}+\frac{m}{2 n}}{m_{1}+m_{2}+m+\frac{m}{n}} .
$$

This gives a good estimation for the position of $G$ if $n$ is sufficiently large. It is clear that $k$ depends on $n$ and if $n \rightarrow \infty$, then $\frac{k}{n}$ tends to the exact value of $A G / d$.

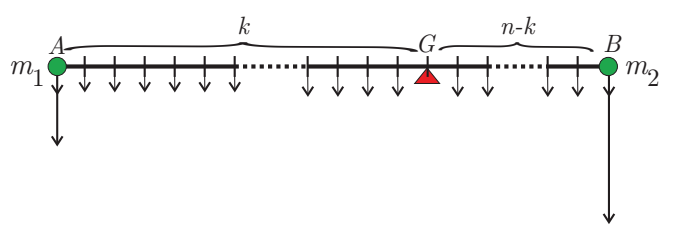

Figure 7. Decompose the rod into small pieces

REMARK 3. The localization of the weight forces for the small pieces can be chosen to act in arbitrary point of the piece. This will influence the value of $\frac{k}{n}$, but not the limit of this expression as $n \rightarrow \infty$. 
$f)$ If we denote by $m_{1}(k)$ and $m_{2}(k)$, the values of the weights in the $k^{t h}$ step, then we have

$$
\left\{\begin{aligned}
& m_{1}(k+1)=m_{1}(k)+\frac{1}{2} \frac{m_{2}(k)-m_{1}(k)}{m_{1}(k)+m_{2}(k)} \cdot m \\
& m_{2}(k+1)=m_{2}(k)+\frac{1}{2} \frac{m_{2}(k)-m_{1}(k)}{m_{1}(k)+m_{2}(k)} \cdot m \\
& \text { where } m_{1}(1)=m_{1} \text { and } m_{2}(1)=m_{2} .
\end{aligned}\right.
$$

Using a computer program (for calculating the terms of these sequences) we can check that this idea is not working. However this idea was not helpful for our initial problem it raises a very interesting question: how to construct the centroid by a recursive algorithm (where one step of the algorithm has some intuitive meaning)? This kind of idea is very useful for treating the general case.

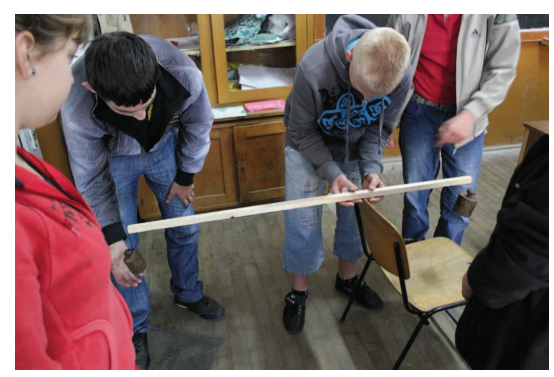

Figure 8. Validating the calculations

Comment 2. We want to focus on the following two aspects:

1. Case $a$ ) shows how the idea of proportionality (which is often used in connection with the calculation of centroid for a system of two points) used without a deeper background can lead to a misconception. A similar problem induced the incorrect ideas $d$ ) and $e$ ).

2. In practice an extra effort is needed in order to make a connection between the abstract notions and tools (centroid, force, moment) and the real objects you are handling. Sometimes this is not an easy task, because in creating the abstract definitions, some aspects were neglected, or restructured, hence you have to clarify what was neglected, what was restructured. On one side this is a major hindering factor in planning IBL activities using advanced mathematical and scientific notions. On the other hand this clarification gives the deepest 
understanding of these notions. This shows that teachers who never taught at this level of understanding, will have serious problems in implementing IBL.

For the second task of Part I the groups discussed all the ideas they formulated (groups C,D also discussed ideas provided by us from previous activities with groups $\mathrm{A}, \mathrm{B}$ ) and made a comparison between the volume of calculations and the complexity of reasoning. From the first sight only $c$ ),g), $h$ ) and $i$ ) seemed to be suitable for obtaining a simple general algorithm/method. $b$ ) and $f$ ) were also analyzed, but only by the groups of university students. For high school students these ideas seemed to be too complicated and for teachers they seemed less teachable.

The shortest and clearest solution was found by extending $i$ ). If $X$ is an exterior point, then the moment of the weight forces $F_{1}, F_{2}, F_{3}$ and $F$ has to equal the moment of the force $F_{1}+F_{2}+F_{3}+F$ localized at the centroid of the system. If we denote $B C=k \cdot d, A C=(1-k) \cdot d, B X=l$ and $B G=x$ (where $G$ is the centroid of the system), we obtain the following equation:

$m_{1} \cdot(l+d)+m_{2} \cdot l+m_{3} \cdot(k \cdot d+l)+m \cdot\left(\frac{d}{2}+l\right)=\left(m+m_{1}+m_{2}+m_{3}\right) \cdot(l+x)$,

hence

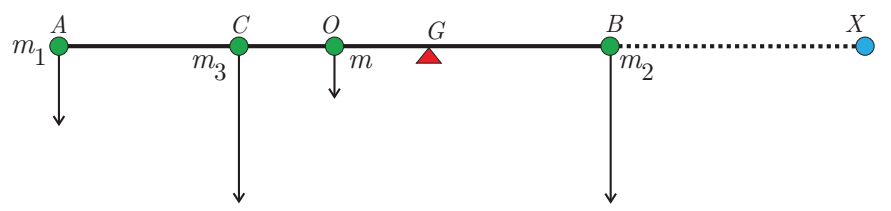

Figure 9. Using an exterior reference point for the moments of the forces

$$
B G=\frac{m_{1}+k \cdot m_{3}+\frac{1}{2} m}{m+m_{1}+m_{2}+m_{3}} d \quad \text { and } \quad A G=\frac{m_{2}+(1-k) \cdot m_{3}+\frac{1}{2} m}{m+m_{1}+m_{2}+m_{3}} d .
$$

REMARK 4. The above formulas (and also the reasoning) show that $X G$ is in fact the weighted arithmetic mean of the distances $X B, X C, X O$ and $X A$ with the weights $m_{2}, m_{3}, m$ and $m_{1}$.

This result shows that $c$ ) is providing the correct answer if we distribute the mass of the rod evenly between $A$ and $B$, but it is no longer about distributing the mass of the rod evenly between all the points $(A, B$ and $C)$. For this reason 
using this idea in other situations may lead to a misconception. On the other hand this gives a hint about why $c$ ) leads to a correct result. In fact the mass of the rod is replaced by two pointwise masses such that the centroid of these two points and the total mass coincide with the centroid and total mass of the rod. This may lead to a series of questions about decomposition of given systems. This is in fact the inverse problem of what we originally posed.

Using $g$ ), we denote $A G=x$. In order to write the moments of the forces we have to deal with two different cases $G \in(A C)$ and $G \in(C B)$. The equations in these cases are equivalent, but the formal argument can be incorrect because if we suppose $G \in(A C)$ and from the obtained value for $x$ we have $G \in(C B)$, then the argument is formally incorrect. In fact this is not a problem, because the equations for the two cases are equivalent, but this has to be clarified. If we use a different notation e.g. $C G=x$, we can obtain a negative value for $x$, which needs an interpretation. This analysis is helpful if we want to use a vectorial approach, but otherwise in the general case (with more weights) can create problems for the students.

Using $h$ ) we have a lot of possibilities. We can construct the centroid $G$ of the system $\left\{A\left(m_{1}\right), B\left(m_{2}\right), O(m)\right\}$ as in the first task and then simply calculate the centroid of the system $\left\{G\left(m_{1}+m_{2}+m\right), C\left(m_{3}\right)\right\}$ or we can use any other partition. If $G_{1}$ and $G_{2}$ are the centroids of the systems $\left\{A\left(m_{1}\right), C\left(m_{3}\right)\right\}$ and $\left\{B\left(m_{2}\right), O(m)\right\}$, then the centroid of the system $\left\{G_{1}\left(m_{1}+m_{3}\right), G_{2}\left(m_{2}+m\right)\right\}$ is the centroid of the initial system. Moreover the given masses can be decomposed in several smaller amounts.

All the previous possibilities need to be experimented in order to understand that if we decompose our system in any possible way into disjoint subsystems, the centroid of the subsystems' centroids is always the centroid of the initial system. For simple systems (having 2, 3 or 4 point masses) we studied all possible decompositions where the initial masses were not decomposed. This was a crucial step for the students in order to formulate a rudimentary version of Archimedes's lemma (see the next remark). In all the cases the comparison of the results can be done by the general formula, by the numeric values or simply by validating the result in practice. At our activities we used all these methods.

REMARK 5. As the most important conclusions we can formulate the definition for the centroid of a system of $n$ collinear points $A_{1}\left(m_{1}\right), \ldots, A_{n}\left(m_{n}\right)$ and the lemma of Archimedes about the centroid of subsystems: 


$$
\text { "tmcs-centroids" — 2013/5/24 — 16:50 — page } 80 \text { — \#14 }
$$

- If the points $A_{1}\left(m_{1}\right), \ldots, A_{n}\left(m_{n}\right)$ are on the same line and $X$ is a fixed, but arbitrary point of this line, then for the centroid $G$ of the system the $\left\{A_{1}\left(m_{1}\right), \ldots, A_{n}\left(m_{n}\right)\right\}$ we have

$$
x_{G}=\frac{m_{1} \cdot x_{A_{1}}+m_{2} \cdot x_{A_{2}}+\cdots+m_{n} \cdot x_{A_{n}}}{m_{1}+m_{2}+\cdots+m_{n}},
$$

where $x_{P}$ denotes the coordinate of the point $P$ with respect to the origin $X$.

- If the system $S=\left\{A_{1}\left(m_{1}\right), \ldots, A_{n}\left(m_{n}\right)\right\}$ is decomposed into disjoint subsystems $S_{1}, S_{2}, \ldots, S_{k}$, whose reunion is $S$ and whose total mass equals with the total mass of $S$, then the centroid of the system formed by the centroids of the subsystems (with the corresponding masses) is the centroid of the initial system $S$.

If $S$ consists of collinear points, the lemma of Archimedes is a direct consequence of the basic properties of operations (commutativity and associativity of summation and distributivity of product with respect to the sum).

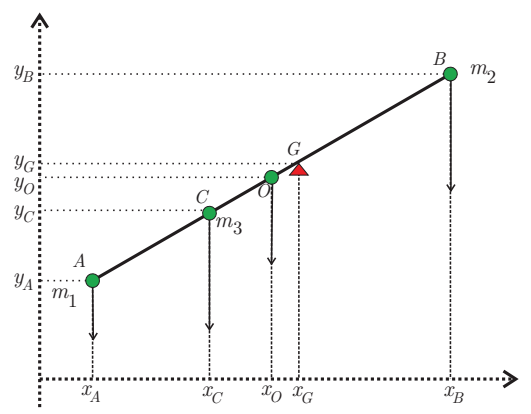

Figure 10. The centroid of a collinear system of points

Comment 3. If we place the points $S=\left\{A_{1}\left(m_{1}\right), \ldots, A_{n}\left(m_{n}\right)\right\}$ in a coordinate system, such that the line $A_{1} A_{2} \ldots A_{n}$ is different from the coordinate axis, we can calculate the coordinates of the centroid in the same way (see Figure 10). If the points are not collinear, we can calculate the coordinates of the centroid by using equivalent systems. Figure 11 shows a possibility to rearrange the points such that the $x$ coordinate of the centroid remains the same. Working with group $\mathrm{C}$, we validated in practice these equivalent transformations during some extra activities, in order to achieve a better understanding of why the coordinates can be treated separately. 

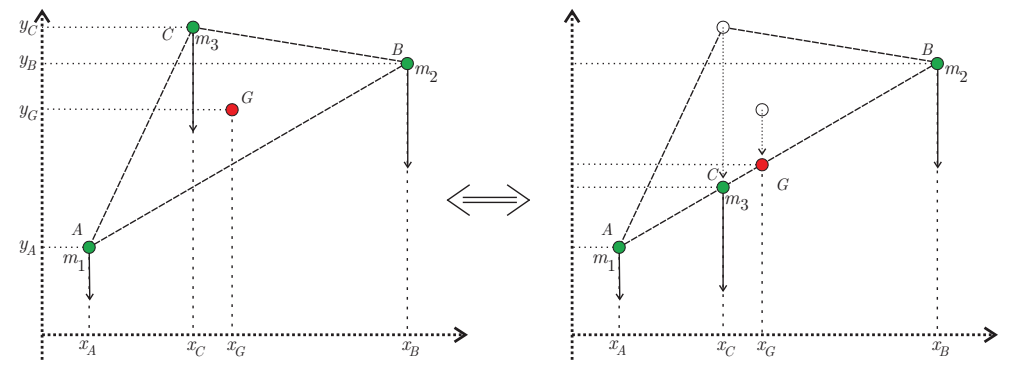

Figure 11. Equivalence in one direction

Comment 4. Looking from the viewpoint of the content, someone might say "Much ado about nothing, these two properties can be presented in half an hour". On the other hand the activity is richer than the content listed above, creates a lot of links to further and deeper understanding and study (to lemma of Archimedes in more general setting, to proofs based on Archimedes lemma, to recursive definition of the centroid, to the abstract definition using integrals, to the understanding of the necessity of a vectorial treatment, etc.). Moreover, such an activity creates a rich context in which student can understand that wellknown pieces of knowledge are rooted in his own thoughts, he can compare the effectiveness of his own ideas with other approaches.

Our experience with students (and also with teachers) is that even if they know all the abstract notions and properties, they rarely can solve the tasks of Part I at the first attempt.

\section{Centroid of polygonal shapes}

The first task of Part II was to determine the centroid of the paper triangle. All participants constructed the centroid of the triangle using one or more medians and they validated their construction with the given tools. On the other hand (as the later analysis pointed out), almost none of them thought about the difference of the triangle and the triangular shaped paper. Based on this achievement all groups constructed the centroid of the quadrilateral shape as the centroid of a quadrilateral. But this time the practice gave an unfavorable verdict, the centroid of a quadrilateral shape is usually not the same as the centroid of the quadrilateral.

This caused a major surprise for teachers and also for students, some of them realized that the argument for triangle was also unfounded. In the next step they 


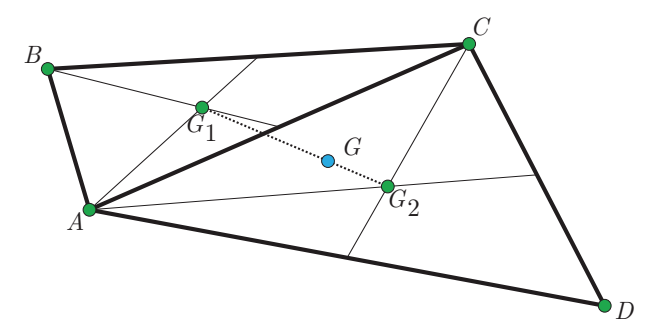

Figure 12. Gvis experimentally determined, $G_{1}$ and $G_{2}$ are the centroids of $A B C$ and $A C D$.

determined experimentally the centroid and tried to give some explanation for their findings. Some groups observed that the centroid of a quadrilateral shape $A B C D$ is on the segment joining the centroids of the triangles $A B C$ and $A C D$ (see Figure 12). This observation allowed to remember the lemma of Archimedes in order to decompose the quadrilateral shape into two triangular shapes and hence to see the centroid $G$ of the quadrilateral shape as the centroid of the system $G_{1}\left(m_{1}\right), G_{2}\left(m_{2}\right)$, where $m_{1}$ and $m_{2}$ are the mass of the triangular shapes $A B C$ and $A C D$. Since the paper (and the board) are (almost) homogeneous, the masses of these triangles are proportional to their area, and this can be calculated by using Heron's formula, if we measure the side lengths of the triangles. This was the solution of several groups. Other groups observed that the quadrilateral can be divided into triangles in two different ways, so the centroid has to be on two different lines. This gives a more elegant construction: if $G_{1}, G_{2}, G_{3}$ and $G_{4}$ are the centroids of the triangles $A B C, A C D, A B D$ and $B D C$, then the centroid of the quadrilateral shape is the intersection point of $G_{1} G_{2}$ and $G_{3} G_{4}$ (see Figure 13). Based on the lemma of Archimedes we can define (and also determine in

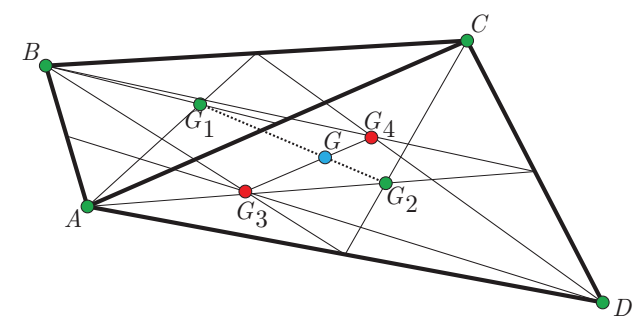

Figure 13. The centroid of a homogeneous quadrilateral domain

practice) the centroid of polygonal shapes and more generally the centroid of 
planar figures, which can be decomposed into simpler figures, whose centroid is known. If $A_{0} A_{1} \ldots A_{n}$ is a convex polygonal shape with $(n+1)$ vertices, the centroid $G$ of this figure is the centroid of the system $G_{n}\left(m_{1}\right), G_{n}^{0}\left(m_{2}\right)$, where $G_{n}$ is the centroid of the polygonal shape $A_{0} A_{1} \ldots A_{n-1}, G_{n}^{0}$ is the centroid of the triangle $A_{0} A_{n} A_{n-1}$, and $m_{1}, m_{2}$ are proportional to the area of the polygon $A_{0} A_{1} \ldots A_{n-1}$ and of the triangle $A_{0} A_{n} A_{n-1}$. Moreover, all the lines $G_{k} G_{k}^{0}$, $0 \leq k \leq n$ constructed in a similar way are concurrent in $G$.

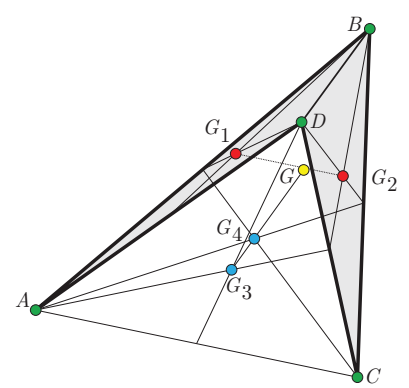

a)

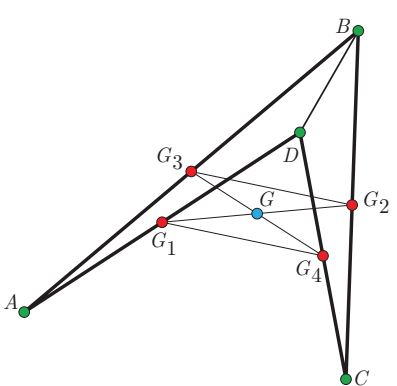

b)

Figure 14. The centroid of a quadrilateral shape and of a quadrilateral

Comment 5. If the polygonal shape $A_{0} A_{1} \ldots A_{n}$ is not convex, we have several options. We can decompose it into smaller convex shapes, or we can add other shapes to it in order to obtain a convex shape. The second method is specially useful in understanding negative weights.

If $A_{0} A_{1} \ldots A_{n}$ is a polygon, the centroid of the system of equally weighted points $\left\{A_{0}, A_{1}, \ldots, A_{n}\right\}$ is called the centroid of the polygon and can be constructed in a similar inductive way. The centroid of the polygon $A_{0} A_{1} \ldots A_{n}$ is the centroid of the system $\left\{G_{n}(n), A_{n}(1),\right\}$ where $G_{n}$ is the centroid of the polygon $A_{0} A_{1} \ldots A_{n-1}$. In Figure 14 we illustrated the construction of the centroid of a concave quadrilateral shape and of a concave quadrilateral, while in Figure 16 the construction of the centroid for a pentagonal shape and a pentagon. On Figure $14 / \mathrm{a}) G_{1}, G_{2}, G_{3}$ and $G_{4}$ are the centroids of the triangle $A B D, B D C$, $A C D$ and $A C B$ respectively, while on Figure $14 / \mathrm{b}) G_{1}, G_{2}, G_{3}$ and $G_{4}$ are the midpoints of the segments $A D, B C, A B$ and $C D$. These figures show that the centroid can lie outside the polygonal shape. In this case the validation of the 
construction in practice is a little bit tricky, because the nail can not support the board in a point which is outside the board. At our activities this problem proved to be a hard one. Only a few participants gave a good solution. Their solution was mainly the same: they constructed a bigger system $S$ using an other board (triangular) and they calculated the centroid of the system $S$ using the centroid of the concave quadrilateral shape.

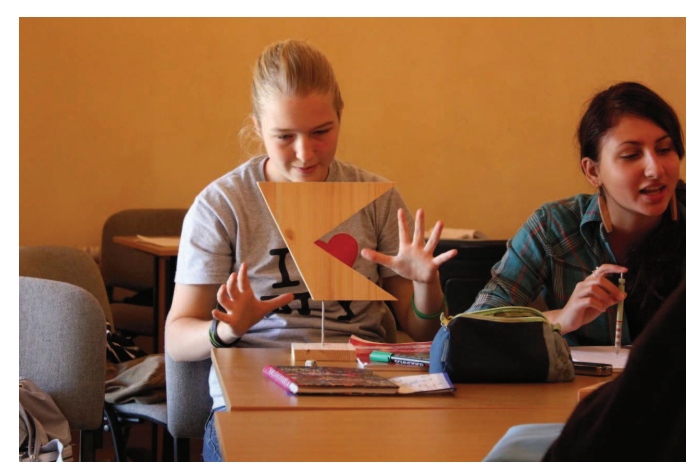

Figure 15. Finding a support point

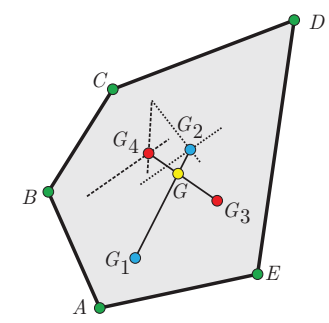

a)

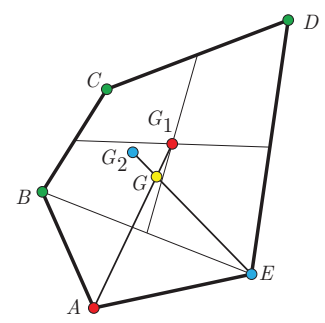

b)

Figure 16. The centroid of a polygonal shape and of a polygon

Comment 6. The previous arguments show that in order to understand the notion of centroid of a polygon and of a polygonal shape (which are usually different) four crucial steps (ingredients) are necessary:

- the centroid of the triangle (which is the same as the centroid of the triangular shape); 


$$
\text { "tmcs-centroids" — 2013/5/24 — 16:50 — page 85 — \#19 }
$$

- the centroid of a quadrilateral and of a quadrilateral shape;

- the lemma of Archimedes;

- the construction of an inductive argument as a defining tool.

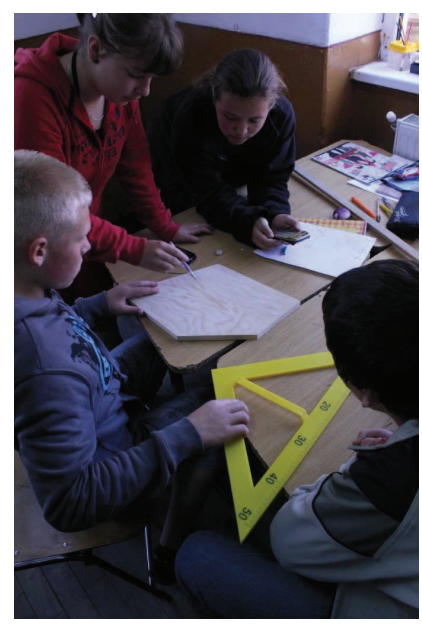

Figure 17. Construction of the centroid for a polygonal shape

Using a similar reasoning the notion of centroid of a system of points can be constructed inductively, adding one point to the previous system in each step. Unfortunately the second and the third key ingredients are not included in the Romanian curricula, the centroid of any planar figure (bounded by curves) was recently removed from the mathematics curricula in the upper secondary, while the center of mass for arbitrary system of points is included in the physics curricula. This is a typical situation: many interdisciplinary IBL activities based on the notions included in the curricula should also handle other notions and properties, which are not included in the curricula.

This proves that in order to include IBL and interdisciplinary approaches into the teaching practice, a strong correlation of the scientific curricula and a deep analysis of the content from an IBL teaching perspective is needed.

At the end of our activities we raised more problems for the participants. We discussed these problems in further sessions. Here we don't detail these discussions or the solution of these problems.

- Find the centroid of the triangle, if the sides are homogeneous rods! 

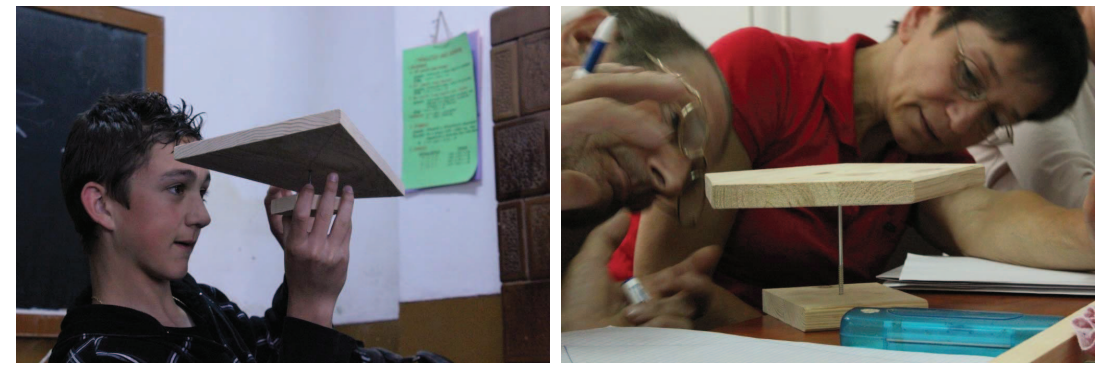

Figure 18. Validation for the centroid of a polygonal shape

- Find the centroid of a polygonal curve, if the sides are homogeneous rods!

- Find the centroid of a triangular board from which we cut out the disc determined by the incircle!

- Find the centroid of a polygonal shape having some weights in different points!

- Design a pentagonal carousel for a group of 5 people!

- Study all the previous problems (those solved during the activity and the proposed ones too) if the weights can be negative!

- ([5]) Five points are given on a circle. A perpendicular is drawn through the centroid of the triangle formed by three of them, to the chord connecting the remaining two. Similar perpendiculars are drawn for each of the remaining nine triplets of points. Prove that the ten lines obtained in this way have a common point!

- (only for teachers) Find applications in geometry for the lemma of Archimedes and for this framework of centroids.

\section{Final conclusions}

1. All the presented ideas and solutions were produced during the activities by the different groups, the authors of this paper only unified and structured them.

2. The participants realized that their ideas can be (and usually are already) incorporated in deep mathematical concepts and properties.

3. Two main hindering factors were identified in relation with planning IBL activities: the differences between theory and practice in some cases (some 
abstract notions need to be "de-abstractified") and the possible IBL gaps in the content.

4. During these activities we could closely track the formation and the clarification of some misconceptions (the evenly distributed mass, the centroid of planar domain). These processes are closely related to IBL.

5. The activity presented here shows how inquiry-based learning is working when highly specialized abstract notions are attacked in practical activities. From this point of view the content itself in this activity is not as important as the processes the students/teachers are going through. On the other hand the content can not be neglected from the point of view of practicing teachers. For this reason in this paper we presented the ideas that appeared at our activities and the final results of some discussions. We do not focus on individual participants, we describe only a general pattern concerning processes. At the beginning all participants (even those from group A) were convinced that the tasks are trivial based on their "theoretical" knowledge. The surprise appeared when nobody succeeded at the first attempt. This created an amount of frustration (is it a tricky rod?, we don't remember the "formulas", we made a mistake during the calculation, etc.) and led to a series of questions and ideas that needed a deeper analysis in order to fit the practice with the calculations. The mathematical content was developed through this analysis. It was very important that there was an easy way (the practice) to validate or to drop each idea and finally to obtain the desired results that were valid in practice, correct in calculations and based on some fundamental theoretical ideas.

\section{Acknowledgements}

This paper is based on the work within the project Primas. Coordination: University of Education, Freiburg. Partners: University of Genève, Freudenthal Institute, University of Nottingham, University of Jaen, Konstantin the Philosopher University in Nitra, University of Szeged, Cyprus University of Technology, University of Malta, Roskilde University, University of Manchester, Babeş-Bolyai University, Sør-Trøndelag University College. The authors wish to thank their students and colleagues attending the training course organized by the BabeşBolyai University in the framework of the FP7 project PRIMAS ${ }^{2}$. The authors 
are grateful to the SimpleX Associaton for the permission of using the teaching materials and for the anonymous referees for their valuable comments.

\section{References}

[1] T. M. Apostol and M. A. Mnatsakanian, Finding Centroids the Easy Way, Math Horizons 8, no. 1 (September 2000), 7-12.

[2] V. I. Arnold, On teaching mathematics, text of the address at the discussion on teaching of mathematics in Palais de Découverte in Paris on 7 March 1997, http://pauli.uni-muenster.de/ munsteg/arnold.html.

[3] I. Caius, Matematică aplicată şi mecanică, Editura Academiei, Bucharest, 1989.

[4] Cs. Csíkos, Problémaalapú tanulás és matematikai nevelés, Vol. 12, Iskolakultúra, 2010.

[5] K. S. Kedlaya, Geometry Unbound, 2006.

[6] S. Laursen, M M.-L. Hassi, M. Kogan, A.-B. Hunter and T. Weston, Evaluation of the IBL Mathematics Project: Student and Instructor Outcomes of Inquiry-Based Learning in College Mathematics, Colorado University, April 2011.

[7] H. Wallberg-Henriksson, V. Hemmo, P. Csermely, M. Rocard, D. Jorde and D. Lenzen, Science education now: a renewed pedagogy for the future of Europe, http://ec.europa.eu/research/science-society/document_library/pdf_06/ report-rocard-on-science-education_en.pdf.

SZILÁRD ANDRÁS

BABEŞ-BOLYAI UNIVERSITY

CLUJ NAPOCA

ROMANIA

E-mail: andraszk@yahoo.com

CSABA TAMÁSI

SIMPLEX ASSOCIATION

MIERCUREA CIUC

ROMANIA

E-mail: tamasics@yahoo.com

(Received May, 2012) 